\title{
Fetal acalvaria with amniotic band syndrome
}

\author{
Suresh Chandran, Meng Keang Lim, Victor Yu-Hei Yu
}

\begin{abstract}
A case of amniotic band syndrome (ABS) presenting with acalvaria is reported. ABS includes a spectrum of non-genetic anomalies, varying from simple digital band constriction to major craniofacial and visceral defects, and even fetal death. Acalvaria is a rare congenital malformation characterised by the absence of the dome-like superior portion of the cranium comprising the frontal, parietal, and occipital bones and dura mater, in the presence of a normal skull base and facial bones with complete cranial contents. No two cases are the same. Acrania or absence of the flat skull bones with disorganised cerebral hemispheres have been reported in the presence of amniotic bands. ABS is an aetiological factor in acalvaria. Appropriate counselling for affected families needs to be given after prenatal diagnosis.

(Arch Dis Child Fetal Neonatal Ed 2000;82:F11-F13)
\end{abstract}

Keywords: acalvaria; acrania; amniotic band syndrome

\section{Case report}

A boy weighing $1510 \mathrm{~g}$, with a head circumference of $30 \mathrm{~cm}$ and a length of $40 \mathrm{~cm}$ at birth, was admitted to the neonatal intensive care unit with a cranial vault defect. He had been a normal vaginal delivery at 32 weeks of gestation after prostaglandin induction, to a healthy gravida 6 para 4 mother, all of whose children are normal. Her medical and obstetric history were unremarkable. She had not used oral contraceptives.

The parents were non-consanguineous, and there was no family history of any congenital anomalies. The mother had regular antenatal care during this pregnancy and tested negative for hepatitis B, toxoplasma, cytomegalovirus and HIV antibodies and was immune to rubella. An antenatal ultrasound scan of the fetus at 24 weeks of gestation showed that the head was shaped abnormally with a lack of gyri over the cerebral hemispheres.

On admission the baby was pink in room air. The calvaria looked like a boggy mass with partial absence of scalp, which was missing from $3 \mathrm{~cm}$ above the ears all around except in the frontal and occipital area where it extended upwards in the midline to varying lengths. The amniotic membrane was fused to the scalp and folded up like a cradle cap; it had entered the placenta (fig 1). Well formed cerebral hemispheres were seen through the amniotic membrane. Palpation confirmed the absence of bony calvaria. Except for the prominence of both eyes, the facial features were normal. The oral cavity was normal. The extremities and spine appeared normal. Systemic examination

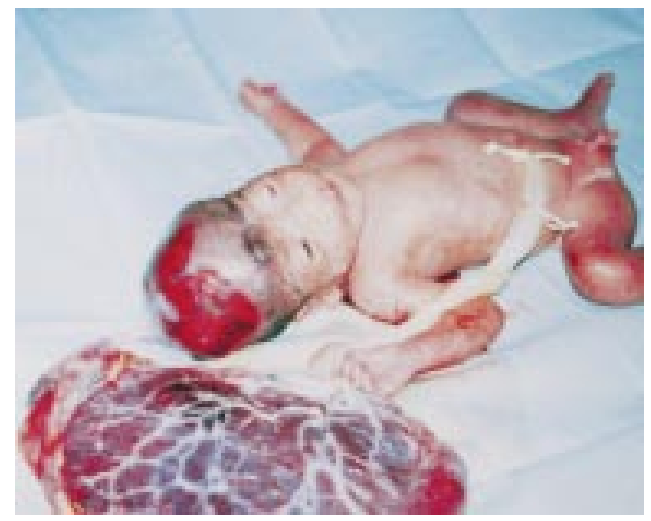

Figure 1 Amniotic band attached to the scalp.

was unremarkable. An $x$-ray picture of the skull revealed partial absence of the frontal, parietal, and occipital bones. An $x$-ray picture of the skeleton showed normal ribs, vertebrae, pelvis and limb bones. A detailed ultrasound study of the abdomen and an echocardiogram were normal. The placenta was intact and weighed $420 \mathrm{~g}$. The baby was given supportive care after appropriate parental counselling and died at 6 hours of age.

\section{Neuropathological examination}

The amniotic membrane had merged with the scalp at varying levels over the calvaria without interruption. The frontal bone terminated 2.5 $\mathrm{cm}$ above the supraorbital plates and the lateral aspects of the parietal bones ended at the level of parietal tuberosity. The occipital bone was absent in the superior aspect. The base of the skull was intact. When the amniotic membrane was opened, a thin shiny transparent membrane could be seen extending over the cerebral hemispheres. Incision of this membrane showed clear fluid and an adherent glistening membrane over the cerebral hemispheres. Fresh blood clots were seen on the hemispheres, probably as a result of birth trauma. There was no ventriculomegaly and the brainstem was intact. Macroscopically, the cerebral cortex, putamen, thalamus, brainstem and cerebellum appeared normal. Histological examination of the brain did not show heterotopia and the neuronal migration pattern was normal. Microscopically, the membranes covering the brain were identified as pia and arachnoid mater.

\section{Discussion}

The prevalence of ABS varies from 1 in 1200 to 1 in 15000 live births, with a slightly higher incidence in women and people of AfroCaribbean descent. ${ }^{1}$ The aetiology of ABS is still obscure. Torpin has cited a genetic aetiology caused by germ plasm defects, calling 
this syndrome "fetal focal dysplasia."' However, this has not been widely accepted as it fails to explain the tremendous variety and asymmetry of lesions involving structures formed at very different times and derived from different germ layers.

Torpin reported that early amnion rupture is the precipitating event. After rupture, the amniotic sac ceases to grow appropriately and gradually separates from the chorion. The amniotic fluid and fetus break through the amnion, coming to rest within the chorion. Transient oligohydramnios ensues. From the chorionic side of the amnion emanate multiple mesoblastic fibrous strings, and these are thought to entangle and entrap the fetal parts. The amnion may roll up into an elasticated rope, stretching across the chorionic cavity and entrapping the fetal head, resulting in faulty migration of the membranous neurocranium which leads to acalvaria. ${ }^{2}$ Most of the subsequent reports on ABS support Torpin's hypothesis. Higginbottom et al reported that deformation, malformation, or disruption results, depending on the time in gestation of the amniotic rupture. ${ }^{3}$ Seeds et al reported incomplete or absent cranial calcification in the spectrum of malformations associated with $\mathrm{ABS}{ }^{4}$ Ossipoff and Hall made a detailed study of the family and gestational histories and found only suggestive evidence that oral contraceptives (when used within 1 month of conception) and abdominal trauma have an aetiological role. ${ }^{5}$ Lockwood et al reported that ABS may not be the consequence of amniotic band formation but rather the result of a multifactorial process responsible for developmental malformations and fetal ectodermal and mesenchymal disruption. Vascular compromise, specifically haemorrhage, may be the central pathogenic feature. ${ }^{6}$

Harris et al described acalvaria as a postneurulation defect. After closure of the anterior neuropore the membranous portion of the neurocranium migrates under the calvarial ectoderm, which subsequently produces the calvarial bones, dura mater, and associated muscles. In acalvaria migration of the membranous neurocranium apparently does not occur. $^{7}$

In the case reported here, the amniotic membrane was well fused to the scalp, and skull bones were absent above the site of attachment of the amniotic membrane. This case supports Torpin's hypothesis of early amnion rupture, with failure of the cranial bones to develop at the site of attachment of the amniotic bands - "early amnion disruption sequence." $2-4$

Prenatal diagnosis of ABS is difficult. When gross fetal abnormalities are detected by ultrasonography or radiography, ABS should be excluded. ${ }^{4}$ Diagnosis of cranial bone defects can be established by ultrasonography early in the second trimester of pregnancy after mineralisation of the skull bones has been completed.

Criteria for diagnosis of acalvaria include absence of calvarial bones with normal development of chondrocranium and presence of cerebral hemispheres. ${ }^{7}$ However, with extensive use of transvaginal sonography, cases of acrania can be diagnosed even in the first trimester of pregnancy if disorganisation of the brain anatomy is also found. Anencephaly and exencephaly, both of which are accompanied by acrania, are the immediate differential diagnosis as facial structures and base of the brain are intact in both. In anencephaly, the brain is absent above the orbit with bulging eyes and a frog-like appearance. Exencephaly is acrania with a large amount of disorganised brain tissue arising from the base of the cranium. Sonographically the outstanding feature is the presence of convolutions or "pseudo sulcal" patterns. Exencephaly is believed to be an embryological precursor of anencephaly. Most cases of acrania eventually progress to anencephaly as a result of slow degeneration of the unprotected brain secondary to mechanical and chemical trauma on exposure to amniotic fluid. ${ }^{8}$ In acalvaria, the usually intact overlying skin protects the brain against this process. ${ }^{7}$ In our case the partial absence of the scalp defect was well covered by the amniotic membrane, affording protection to the underlying brain.

It is important to differentiate acalvaria from acrania in utero by sonography as the brain can be normal and potentially treatable in the former, but in the latter most of these progress to anencephaly. Ultrasonography did not indicate calvarial defects in the reported case, which could be explained by the diagnostic inconsistency seen in ABS with craniofacial defects. ${ }^{4}$ Severe osteogenesis imperfecta or congenital hypophosphatasia may cause inadequate visualisation or ossification of the calvaria, generating an erroneous diagnosis of acalvaria. ${ }^{9}$

Neonatal diagnosis of ABS is frequently difficult and is accurately diagnosed in only $29 \%$ to $50 \%$ of cases. Five per cent of anencephalic babies have ABS. The presence of fibrous bands at constriction points is helpful in the diagnosis. Craniofacial deformities in ABS are typically often bizarre and frequently nonembryological in location. Internal organ anomalies in infants with ABS are rare..$^{3-5}$

No chromosomal abnormalities have been reported with acalvaria and most of the reported cases of ABS have presented as sporadic events in otherwise normal families. ${ }^{4}$ It is appropriate to counsel the parents explicitly about the non-genetic nature of these malformations. ${ }^{7-10}$ Following ultrasonographic diagnosis early in the second trimester, termination of pregnancy should be offered because acalvaria is fatal.

With a morphologically and histologically normal brain in acalvaria, as reported in this case, future developments in surgical repair of bone abnormalities will improve the prognosis of this unique anomaly.

1 Garza A, Cordero JF, Mulinare J. Epidemiology of the early amnion rupture spectrum of defects. Am $\mathcal{F}$ Dis Child 1988;142:54-14.

2 Torpin R. Fetal malformations caused by amnion rupture during gestation. First Edn. Springfield, Ill: Charles C Thomas, 
3 Higginbottom MC, Jones KL, Hall BD, Smith DW. The Amniotic band disruption complex: Timing of amniotic rupture and variable spectra of consequent defects. F Pediatr 1979;95:544-9.

4 Seeds JW, Cefalo RC, Herbert WNP. Amniotic band syndrome Am f Obstet Gynecol 1982;144:243-8.

Ossipoff V, Hall BD. Etiologic factors in the amniotic band syndrome: study of 24 patients. Birth Defects 1977;13:117-32.

6 Lockwood C, Ghidini A, Romero R, Hobbins JC. Amniotic band syndrome: Re-evaluation of its pathogenesis. $A m \mathcal{F}$ Obstet Gynecol 1989;160:1030-3.
7 Harris CP, Townsend JJ, Carey JC. Acalvaria: A unique congenital anomaly. Am f Med Genet 1993;46:694-9.

8 Hendricks SK, Cyr DR, Nyberg DA, et al. Exencephalyclinical and ultrasonic correlation to anencephaly. Obstet Gynecol 1988;72:898-900

9 Weissman A, Diukman R, Auslender R. Fetal acrania: 5 new cases and review of the literature. F Clin Ultrasound 1997;25:511-14.

10 Foulkes GD, Reinker K. Congenital constriction band syndrome: A seventy -year experience. $\mathcal{F}$ Pediatr Orthop 1994;14:242-8.

\section{STAMPS IN NEONATOLOGY}

\section{The Patient's Tale}

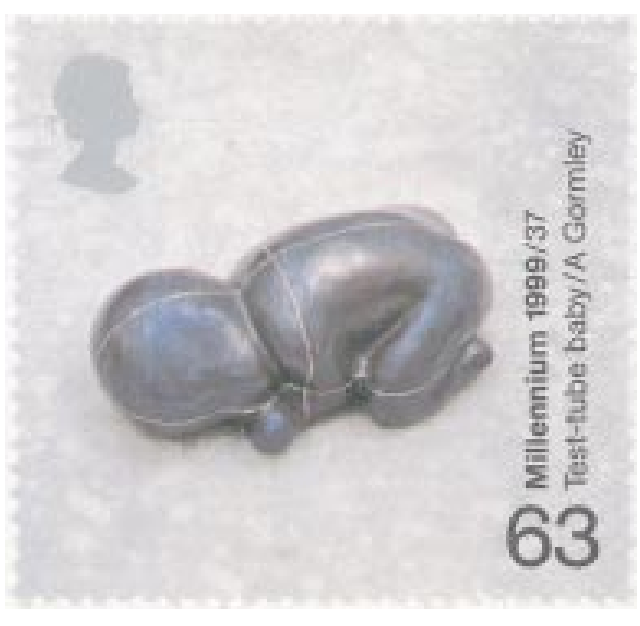

Each month during 1999 the Royal Mail issued a set of four stamps to celebrate the millennium. Each issue has a theme or historical "tale" including the Inventor's Tale (January 1999), the Worker's Tale (May), and the Scientist's Tale (August). Four stamps appeared on 2 March 1999 for the Patient's Tale. The 20 p value marked Edward Jenner's achievements in vaccination, the $26 \mathrm{p}$ value nursing care, and the $43 \mathrm{p}$ value Alexander Fleming's discovery of penicillin. The highest value in the set and number 37 in the series of 48 is illustrated and commemorates the developments leading to the test tube baby.

M K DAVIES Consultant Cardiologist, Selly Oak Hospital, Birmingham, UK A J MAYNE

Consultant Paediatrician, Sandwell Hospital, Birmingham, UK 